\title{
Nonmaximally entangled states can be better for quantum correlation distribution and storage
}

\author{
Xin-Wen Wang, ${ }^{1,2, *}$ Shi-Qing Tang, ${ }^{1}$ Ji-Bing Yuan,${ }^{1}$ and Le-Man Kuang ${ }^{1, \dagger}$ \\ ${ }^{1}$ Department of Physics and Key Laboratory of Low-Dimensional Quantum Structures and Quantum Control of Ministry of Education, \\ Hunan Normal University, Changsha 410081, People's Republic of China \\ ${ }^{2}$ Department of Physics and Electronic Information Science, \\ Hengyang Normal University, Hengyang 421002, People's Republic of China
}

\begin{abstract}
For carrying out many quantum information protocols entanglement must be established in advance between two distant parties. Practically, inevitable interaction of entangled subsystems with their environments during distribution and storage will result in degradation of entanglement. Here we investigate the decoherence of twoqubit entangled states in the local amplitude noise. We show that there exists a set of partially entangled states that are more robust than maximally entangled states in terms of the residual quantum correlation measured by concurrence, fully entangled fraction, and quantum discord. This result indicates that nonmaximally entangled states can outperform maximally entangled states for quantum correlation distribution and storage under the amplitude damping. It also educes a notable consequence that the ordering of states under quantum correlation monotones can be reversed even by local trace-preserving and completely positive maps.
\end{abstract}

PACS numbers: 03.67.Hk, 03.65.Yz, 03.67.Pp

Keywords: Quantum correlation, decoherence, concurrence, fully entangled fraction, quantum discord

Establishing quantum entanglement between two distant parties is a prerequisite for many fundamental tests of quantum theory and numerous protocols in long-range quantum information processing [1]. There are three general ways for providing long-distance entanglement. One is to prepare a bipartite entangled pure state in a server and then physically send two subsystems to two distant parties, respectively. Note that the entanglement of distributed flyingsystems could be transferred to two static systems [2]. The second relies on the successive interactions of a carrier quantum system with two distant particles [3]. The third is based on the interference of two photons emitted, respectively, from two distant massive qubits [4]. All these methods are usually utilized to create entanglement between two quantum repeaters or two chosen nodes of a quantum network [4, 5].

The above scenarios involve entanglement distribution and storage. During these processes the entangled systems unavoidably interact with their environments, respectively, which results in degradation of established entanglement between the two parties. Investigating the robustness of entangled states in noise environments may help to gain some insight into the properties of decoherence and entanglement, which will provide useful hints for maintaining entanglement. Many efforts have been devoted to the study of dynamics of entanglement or quantum correlation for a given initial two-qubit entangled state in various environments (see, e.g., [6]). In this Brief Report, we are going to focus on the question of maximal residual entanglement or quantum correlation of two-qubit cat-like states for given local noises and decoherence strengths. It has been shown that the residual entanglement is proportional to the initial entanglement of the cat-like state in the case of one-side noise [7], which implies that the maximal residual entanglement is attained iff the initial state is maximally entangled. The purpose of this Brief Report is to explicitly demonstrate the counterintuitive phenomenon in the case of two-side noises.

The noise environment considered in this work is the amplitude-damping environment [8]. Amplitude-damping

*xwwang@mail.bnu.edu.cn

${ }^{\dagger}$ lmkuang@hunnu.edu.cn decoherence is applicable to many practical qubit systems, including vacuum-single-photon qubit with photon loss, atomic qubit with spontaneous decay, and superconducting qubit with zero-temperature energy relaxation. The action of an amplitude-damping environment $\mathcal{E}$ on a single qubit state $\varrho$ can be described as

$$
\varrho \rightarrow \mathcal{E}(\varrho)=M_{0} \varrho M_{0}^{+}+M_{1} \varrho M_{1}^{+},
$$

where $M_{0}$ and $M_{1}$ are the Krauss operators defined by

$$
M_{0}=\left(\begin{array}{cc}
1 & 0 \\
0 & \sqrt{\bar{d}}
\end{array}\right), \quad M_{1}=\left(\begin{array}{cc}
0 & \sqrt{d} \\
0 & 0
\end{array}\right)
$$

with $0 \leqslant d \leqslant 1$ and $\bar{d}=1-d$. The amplitude-damping environment is trace preserving, that is, $\sum_{j=0,1} M_{j}^{+} M_{j}=I$. Note that $d=0$ denotes the noise-free case. For $d=1$, the state $\mathcal{E}(\varrho)$ reduces to a classical state. Therefore, throughout this Brief report, we only consider the decoherence strength $0<d<1$.

We use three different measures, that is, concurrence [9], fully entangled fraction (FEF) [11], and quantum discord (QD) [12], to quantify the quantum correlation of the decoherent state evolving from the initial entangled state under the action of amplitude-damping environment. Concurrence is related straightforwardly to the entanglement of formation and has been considered as a dependable measure of entanglement for two-qubit entangled states [9]. FEF, which expresses the purity of a mixed state $\rho$, plays a central role in quantum teleportation and entanglement distillation [11, 13], and may show different properties from concurrence [14]. QD has been regarded as a more general measure of nonclassical correlations than entanglement and even survives entanglement [12], and has been proposed as the key resource present in certain quantum computational models and quantum communication tasks without containing much entanglement [15]. We show that each of the three measures reaches its maximum value when the initial two-qubit pure state is a nonmaximally entangled state but not maximally entangled state. More importantly, there exists a set of partially entangled states, and when the initial state belongs to this set, all the aforementioned quantities of the decoherent state are larger than that in the case where the initial state is maximally entangled. That is to say, some partially entan- 
gled states can outperform maximally entangled states for acting as the initial state.

Supposing that two qubits are initially in an entangled state

$$
|\psi\rangle=\sqrt{u}|0\rangle|0\rangle+\sqrt{\bar{u}} e^{i \varphi}|1\rangle|1\rangle,
$$

where $0 \leqslant u \leqslant 1, \bar{u}=1-u$, and $\{|0\rangle,|1\rangle\}$ denotes the computational basis. The entanglement of $|\psi\rangle$ can be quantified by concurrence. For a two-qubit state $\rho$ the concurrence is given by $C(\rho)=\max \left\{0, \sqrt{\omega_{1}}-\sqrt{\omega_{2}}-\sqrt{\omega_{3}}-\sqrt{\omega_{4}}\right\}$, where $\left\{\omega_{i}\right\}$ are the eigenvalues of $\rho\left(\sigma_{y} \otimes \sigma_{y} \rho^{*} \sigma_{y} \otimes \sigma_{y}\right)$ in decreasing order with $\rho^{*}$ the complex conjugation of $\rho$ and $\sigma_{y}$ the conventional Pauli matrix [9]. After a simple calculation one can obtain $\mathcal{C}(|\psi\rangle)=2 \sqrt{\bar{u} u}$. Obviously, $\mathcal{C}(|\psi\rangle)$ is equal to unit if $u=1 / 2$, which means that $|\psi\rangle$ is a maximally entangled state, denoted by $\left|\psi_{m}\right\rangle$, in this case. We now consider that each qubit undergoes an amplitudedamping decoherence during transmitting or storing. The maximally entangled state $\left|\psi_{m}\right\rangle$ was usually utilized to implement many quantum information protocols, e.g., quantum teleportation [10]. The following discussions suggest that $|\psi\rangle$ with $u \neq 1 / 2$ could be better than $\left|\psi_{m}\right\rangle$ for performing these tasks in the amplitude-damping environment. For convenience in analysis, we assume the decoherence strengths of two qubits are the same (which makes no difference to the final conclusion). Then the state $|\psi\rangle$ is degraded into

$$
\begin{aligned}
\rho^{d, u} & =\sum_{j, k=0,1} M_{j} \otimes M_{k}|\psi\rangle\langle\psi| M_{j}^{+} \otimes M_{k}^{+} \\
& =\left(\begin{array}{cccc}
u+\bar{u} d^{2} & 0 & 0 & \bar{d} \sqrt{u \bar{u}} e^{-i \varphi} \\
0 & \bar{u} \bar{d} d & 0 & 0 \\
0 & 0 & \bar{u} \bar{d} d & 0 \\
\bar{d} \sqrt{u \bar{u}} e^{i \varphi} & 0 & 0 & \bar{u} \bar{d}^{2}
\end{array}\right),
\end{aligned}
$$

where $d$ denotes the decoherence strength of each qubit in the noise environment. The superscripts of $\rho$ denote that the state $\rho$ is dependent on the parameters $d$ and $u$.

The concurrence of $\rho^{d, u}$ is given by

$$
\mathcal{C}\left(\rho^{d, u}\right)=\max \{0,2 \bar{d}(\sqrt{\bar{u} u}-\bar{u} d)\} .
$$

It can be seen that $\mathcal{C}\left(\rho^{d, u}\right)=0$ if $\sqrt{u / \bar{u}}<d$, which means the occurrence of entanglement sudden death [16]. For a given $d$, the maximum value of $\mathcal{C}\left(\rho^{d, u}\right)$ is

$$
\mathcal{C}\left(\rho^{d, u=u_{m}}\right)=\bar{d}\left(\sqrt{1+d^{2}}-d\right)
$$

with

$$
u_{m}=\frac{1}{2}+\frac{d}{2 \sqrt{1+d^{2}}} .
$$

$\mathcal{C}\left(\rho^{d, u=1 / 2}\right)<\mathcal{C}\left(\rho^{d, u=u_{m}}\right)$ implies an interesting phenomenon that the decoherent state $\rho^{d, u}$ has the maximal amount of entanglement if the initial state $|\psi\rangle$ is an appropriate partially entangled state but not maximally entangled state. This result is different from that in the case of one-side noise where the decoherent state has the maximal amount of entanglement in terms of concurrence when the initial state is maximally entangled (i.e, $u=1 / 2$ ) [7, 14]. Particularly, if

$$
\frac{1}{2}<u=u^{\prime}<\frac{1}{2}+\frac{d}{1+d^{2}}
$$

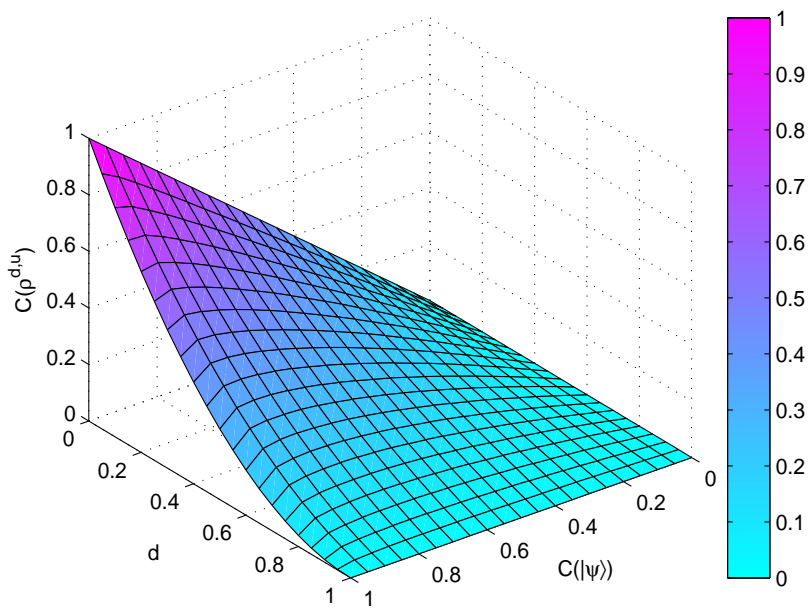

FIG. 1: (Color online) The residual entanglement $\mathcal{C}\left(\rho^{d, u}\right)$ versus the initial entanglement $\mathcal{C}(|\psi\rangle)$ for $1 / 2 \leqslant u \leqslant 1$.

the corresponding concurrence is larger than that in the case of $u=1 / 2$, that is, $\mathcal{C}\left(\rho^{d, u^{\prime}}\right)>\mathcal{C}\left(\rho^{d, 1 / 2}\right)$. The above result implies that the amount of entanglement of $\rho^{d, u}$ is not monotonic with respect to that of $|\psi\rangle$. For showing this more clearly, we rewrite $\mathcal{C}\left(\rho^{d, u}\right)$ as a function of $\mathcal{C}(|\psi\rangle)$,

$$
\begin{aligned}
\mathcal{C}\left(\rho^{d, u}\right)= & \bar{d}\left[C(|\psi\rangle)-d\left(1-\sqrt{1-C(|\psi\rangle)^{2}}\right)\right] \\
& \text { for } \frac{1}{2} \leqslant u \leqslant 1 \\
\mathcal{C}\left(\rho^{d, u}\right)= & \max \left\{0, \bar{d}\left[C(|\psi\rangle)-d\left(1+\sqrt{1-C(|\psi\rangle)^{2}}\right)\right]\right\} \\
& \text { for } 0 \leqslant u<\frac{1}{2} .
\end{aligned}
$$

Figure 1 shows that when $1 / 2 \leqslant u \leqslant 1, \mathcal{C}\left(\rho^{d, u}\right)$ is indeed non-monotonic with respect to $\mathcal{C}(|\psi\rangle)$ for an arbitrarily given $d$. In fact, $\mathcal{C}\left(\rho^{d, u}\right)$ takes the extremum $\mathcal{C}\left(\rho^{d, u_{m}}\right)$ on condition that $\mathcal{C}(|\psi\rangle)$ is equal to $1 / \sqrt{1+d^{2}}$ but not one.

FEF, another important entanglement measure, may behave differently from concurrence. For example, if the concurrence of a mixed state $\rho_{1}$ is larger than the concurrence of a mixed state $\rho_{2}$, one cannot conclude that the FEF of $\rho_{1}$ is also larger than the FEF of $\rho_{2}$ [14]. FEF is defined as the maximum overlap of the state $\rho$ with a maximally entangled state,

$$
\mathcal{F}(\rho)=\max _{|\phi\rangle}\langle\phi|\rho| \phi\rangle
$$

with the maximization taken over all maximally entangled states $|\phi\rangle$. If and only if $\mathcal{F}(\rho)$ is larger than $1 / 2$, quantum teleportation can exhibit its superiority over the classical strategy and entanglement distillation can be carried out effectively in the protocols based on the resource state $\rho[11,13]$. As a matter of fact, the larger the FEF $\mathcal{F}(\rho)$ is, the higher teleportation fidelity and entanglement distillation efficiency can be achieved [11, 13]. For two-qubit systems $\mathcal{F}(\rho)$ can be analytically expressed by $[17$

$$
\mathcal{F}(\rho)=\frac{1+\nu_{1}+\nu_{2}-\operatorname{sgn}[\operatorname{det}(\tilde{T})] \nu_{3}}{4}
$$


where $\left\{\nu_{i}\right\}$ are the decreasingly ordered singular values of the $3 \times 3$ real matrix $\tilde{T}=\left[\operatorname{Tr}\left(\rho \sigma_{i} \otimes \sigma_{j}\right)\right]_{3 \times 3}$ with $\left\{\sigma_{i}, i=\right.$ $1,2,3\}$ the Pauli matrices and $\operatorname{sgn}[\operatorname{det}(\tilde{T})]$ is the sign of the determinant of $\tilde{T}$. In our case, the FEF can be calculated to be

$$
\mathcal{F}\left(\rho^{d, u}\right)=\frac{1}{2}+\bar{d}(\sqrt{\bar{u} u}-\bar{u} d) .
$$

It can be easily verified that $\mathcal{F}\left(\rho^{d, u}\right)$ gives the maximum

$$
\mathcal{F}\left(\rho^{d, u=u_{m}}\right)=\frac{1}{2}+\frac{\bar{d}}{2}\left(\sqrt{1+d^{2}}-d\right)
$$

and that $\mathcal{F}\left(\rho^{d, u^{\prime}}\right)>\mathcal{F}\left(\rho^{d, 1 / 2}\right)$. However, FEF is not an entanglement monotone as it may increase under tracepreserving local operations and classical communication (TPLOCC) for mixed states [17-19]. This leads to the advent of the concept of the maximum achievable FEF given by

$$
\mathcal{F}^{*}(\rho)=\max _{\text {TPLOCC }} \mathcal{F}(\rho) \geqslant \mathcal{F}(\rho)
$$

for any $2 \otimes 2$ density matrix $\rho$ [19]. $\mathcal{F}^{*}(\rho)$ was shown to be an entanglement monotone [19]. Thus $\mathcal{F}\left(\rho^{d, 1 / 2}\right)<\mathcal{F}\left(\rho^{d, u^{\prime}}\right)$ does not necessarily lead to $\mathcal{F}^{*}\left(\rho^{d, 1 / 2}\right)<\mathcal{F}^{*}\left(\rho^{d, u^{\prime}}\right)$. For a mixed state of two qubits $\rho$, it has been proved that [20]

$$
\mathcal{F}^{*}(\rho) \leqslant \frac{1+\mathcal{N}(\rho)}{2},
$$

where $N(\rho)$ denotes the negativity [21] of $\rho$ given by $\mathcal{N}(\rho)=\max \left\{0,-2 \lambda_{m}\right\}$ with $\lambda_{m}$ the minimal eigenvalue of the partial transpose of $\rho$ denoted as $\rho^{\Gamma}$. The equality is achieved iff the eigenvector corresponding to the negative eigenvalue of $\rho^{\Gamma}$ is maximally entangled [20], and this condition is equivalent to the condition $\mathcal{N}(\rho)=\mathcal{C}(\rho)$. In our case,

$$
\mathcal{F}^{*}\left(\rho^{d, u}\right)=\mathcal{F}\left(\rho^{d, u}\right)=\frac{1+\mathcal{N}\left(\rho^{d, u}\right)}{2}
$$

if $\sqrt{u / \bar{u}} \geqslant d$ corresponding to $\mathcal{F}\left(\rho^{d, u}\right) \geqslant 1 / 2$. Note that for the values of $u$ given in Eq. (8) the condition $\sqrt{u / \bar{u}} \geqslant d$ is naturally satisfied. In these cases, therefore, there is no benefit from local processing of the state $\rho^{d, u}$. In other words, TPLOCC is not required for obtaining the maximum achievable FEF. Now, we can safely conclude that $\mathcal{F}^{*}\left(\rho^{d, u^{\prime}}\right)>\mathcal{F}^{*}\left(\rho^{d, 1 / 2}\right)$ and $\mathcal{F}^{*}\left(\rho^{d, u}\right)$ gives the extremum $\mathcal{F}^{*}\left(\rho^{d, u_{m}}\right)=\mathcal{F}\left(\rho^{d, u_{m}}\right)$ when the initial state $|\psi\rangle$ is a partially entangled state with $u=u_{m}$. In fact, $\mathcal{F}^{*}\left(\rho^{d, u}\right)$ has the same behavior as $\mathcal{C}\left(\rho^{d, u}\right)$ for the case $\sqrt{u / \bar{u}} \geqslant d$.

QD is a more general measure of nonclassical correlation than entanglement [12] and may exhibit different features from concurrence and FEF. Thus, it is necessary to investigate the QD of the decoherent state $\rho^{d, u}$ in Eq. (4). For a given quantum state $\rho^{A B}$ of a composite bipartite system $A B$, QD is defined as the difference of the quantum mutual information and the classical correlation [12]. The quantum mutual information is given by $\mathcal{I}\left(\rho^{A B}\right)=S\left(\rho^{A}\right)+$ $S\left(\rho^{B}\right)-S\left(\rho^{A B}\right)$, where $S(\rho)=-\operatorname{Tr}\left(\rho \log _{2} \rho\right)$ denotes the von Neumann entropy and $\rho^{A}\left(\rho^{B}\right)$ is the reduced density matrix for subsystem $A(B)$. The classical correlation is quantified by $\mathcal{J}_{A}\left(\rho^{A B}\right)=S\left(\rho^{B}\right)-\min _{E_{k}^{A}} \sum_{k} p_{k} S\left(\rho^{B \mid k}\right)$,
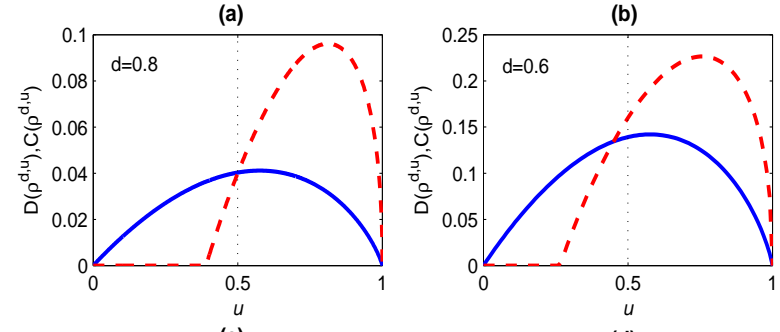

(c)
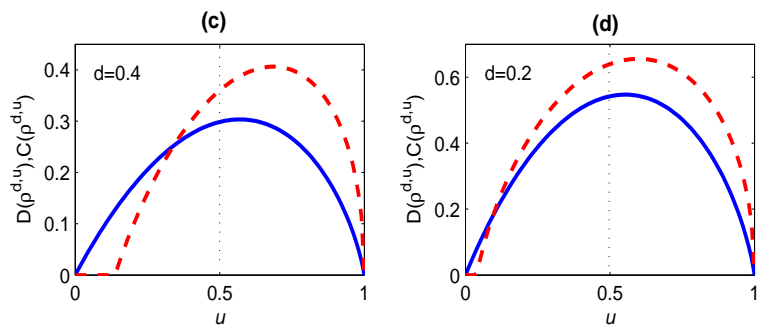

FIG. 2: (Color online) The concurrence $\mathcal{C}\left(\rho^{A B}\right)$ (dashed and red line) and the $\mathrm{QD} \mathcal{D}\left(\rho^{A B}\right)$ (solid and blue line) versus the parameter $u$ of the initial state for a given decoherence strength $d$. (a) $d=0.8$; (b) $d=0.6$; (c) $d=0.4 ;$ (d) $d=0.2$.

where the minimum is taken over all possible positive operator valued measures (POVMs) or von Neumann measurements $\left\{E_{k}^{A}\right\}$ on subsystem $A$ with $p_{k}=\operatorname{Tr}\left(E_{k}^{A} \rho^{A B}\right)$ and $\rho^{B \mid k}=\operatorname{Tr}_{A}\left(E_{k}^{A} \rho^{A B}\right) / p_{k}$. Then QD is given by $\mathcal{D}_{A}\left(\varrho^{A B}\right)=\mathcal{I}\left(\rho^{A B}\right)-\mathcal{J}_{A}\left(\rho^{A B}\right)$. Generally, it is very difficult to analytically compute the classical correlation $\mathcal{J}_{A}\left(\rho^{A B}\right)$ due to the difficulty of finding the optimal measurement for minimizing $\sum_{k} p_{k} S\left(\rho^{B \mid k}\right)$. Fortunately, the state $\rho^{d, u}$ in Eq. (4) is a particular case of $\mathrm{X}$-states whose QDs have been intensely investigated [22, 23]. For a real X-state

$$
X^{A B}=\left(\begin{array}{cccc}
\rho_{00} & 0 & 0 & \rho_{03} \\
0 & \rho_{11} & \rho_{12} & 0 \\
0 & \rho_{12} & \rho_{22} & 0 \\
\rho_{03} & 0 & 0 & \rho_{33}
\end{array}\right)
$$

with $\left|\rho_{12}+\rho_{03}\right| \geqslant\left|\rho_{12}-\rho_{03}\right|$, it has been proved [23] that the optimal measurement for $\mathcal{D}_{A}\left(X^{A B}\right)$ is $\sigma_{z}^{A}$ if $\left(\left|\rho_{12}\right|+\right.$ $\left.\left|\rho_{03}\right|\right)^{2} \leqslant\left(\rho_{00}-\rho_{11}\right)\left(\rho_{33}-\rho_{22}\right)$ or $\sigma_{x}^{A}$ if $\mid \sqrt{\rho_{00} \rho_{33}}-$ $\sqrt{\rho_{11} \rho_{22}}|\leqslant| \rho_{12}|+| \rho_{03} \mid$. Via a local unitary transformation, which preserves the $\mathrm{QD}$, the complex factors $e^{ \pm i \varphi}$ in the state $\rho^{d, u}$ can be directly removed and $\rho^{d, u}$ is transformed into a real X-state. Therefore, we can drop the factors $e^{ \pm i \varphi}$ when we evaluate the $\mathrm{QD} \mathcal{D}\left(\rho^{d, u}\right)$, and the theorem above is applied to the state $\rho^{d, u}$. Then it can be easily verified that $\rho^{d, u}$ satisfies the latter condition in the theorem and the optimal measurement for $\mathcal{D}\left(\rho^{d, u}\right)$ is $\sigma_{x}$. After a straightforward calculation we obtain the QD of such a state

$$
\mathcal{D}\left(\rho^{d, u}\right)=\sum_{i=1}^{4} \lambda_{i} \log _{2} \lambda_{i}-\sum_{j=5}^{8} \lambda_{j} \log _{2} \lambda_{j},
$$

where

$$
\begin{aligned}
& \lambda_{1,2}=\bar{u} \bar{d} d \\
& \lambda_{3,4}=\frac{1}{2}(1-2 \bar{u} \bar{d} d) \pm \frac{1}{2} \sqrt{1-4 \bar{u} \bar{d} d}, \\
& \lambda_{5,6}=\frac{1}{2} \pm \frac{1}{2} \sqrt{1-4 \bar{u} \bar{d} d}, \\
& \lambda_{7}=u+\bar{u} d, \quad \lambda_{8}=\bar{u} \bar{d} .
\end{aligned}
$$


Here we have dropped the qubit index in $\mathcal{D}\left(\rho^{d, u}\right)$ because $\rho^{d, u}$ is invariant under permutation of the two qubits. Figure 2 shows that for a given $d$ it does not occur in the case $u=$ $1 / 2$ that $\mathcal{D}\left(\rho^{d, u}\right)$ reaches the extremum. This indicates that if the initial state $|\psi\rangle$ is a suitable partially entangled state $(u \neq 1 / 2)$, the QD $\mathcal{D}\left(\rho^{d, u}\right)$ will attain the maximum value.

As shown above, the concurrence, maximum achievable FEF, and QD of the decoherent state $\rho^{d, u}$ in Eq. (4) have the same feature that each of them attains its maximum value iff the initial state $|\psi\rangle$ is a suitable partially entangled state. That is, $\mathcal{C}\left(\rho^{d, u}\right), \mathcal{F}^{*}\left(\rho^{d, u}\right)$, and $\mathcal{D}\left(\rho^{d, u}\right)$ achieve their extrema iff $u$ is equal to $u_{1}=u_{m} \neq 1 / 2, u_{2}=u_{m}$, and $u_{3} \neq 1 / 2$, respectively. It is worth pointing out that for different values of $d$ different optimal values of concurrence, FEF, and QD are obtained, respectively. The corresponding partially entangled states are different as well. Furthermore, for $u^{\prime} \in\left(1 / 2, u_{0}^{\prime}\right)$ and $u^{\prime \prime} \in\left(1 / 2, u_{0}^{\prime \prime}\right)$ we have $\mathcal{C}\left(\rho^{d, u^{\prime}}\right)>\mathcal{C}\left(\rho^{d, 1 / 2}\right), \mathcal{F}^{*}\left(\rho^{d, u^{\prime}}\right)>\mathcal{F}^{*}\left(\rho^{d, 1 / 2}\right)$, and $\mathcal{D}\left(\rho^{d, u^{\prime \prime}}\right)>\mathcal{D}\left(\rho^{d, 1 / 2}\right)$. Note that $u_{1}=u_{2} \neq u_{3}$ and $u_{0}^{\prime} \neq u_{0}^{\prime \prime}$ (see Fig. 2), which provides a new example of showing the different behaviors of QD and entanglement. The above results indicate that if the initial state $|\psi\rangle$ belongs to a suitable set of partially entangled states, all the concurrence, maximum achievable FEF, and QD of the decoherent state $\rho^{d, u}$ are larger than that in the case where $|\psi\rangle$ is maximally entangled. Therefore, we conclude that partially entangled states can outperform maximally entangled states for acting as the initial entangled state. It is easy to verify that all the concurrence, maximum achievable FEF, and QD of the initial state $|\psi\rangle$ reach their maximum values when $u=1 / 2$. Thus the results obtained above also imply that the ordering of states under concurrence, maximum achievable FEF, and QD can be reversed, respectively, even though the states undergo identical local interactions via trace-preserving and completely positive maps.

In conclusion, we have investigated the robustness of twoqubit cat-like states in the amplitude-damping environment. We demonstrated that nonmaximally entangled states can be better than maximally entangled states for quantum correlation distribution and storage. The obtained results may be of practical importance for quantum information processing as well as contribute to our understanding of quantum noises and quantum correlations. By the way, it has also been reported that nonmaximally entangled states can outperform maximally entangled states for several quantum tasks [24].

\section{Acknowledgments}

This work was supported by the NSFC (No. 11004050, No. 11075050, and No. 11375060), the Key Project of Chinese Ministry of Education (No. 211119), and the China Postdoctoral Science Foundation funded project (No. 2013T60769).
[1] R. Horodecki, et al., Rev. Mod. Phys. 81, 865 (2009); J. W. Pan, et al., ibid. 84, 777 (2012).

[2] B. Kraus and J. I. Cirac, Phys. Rev. Lett. 92, 013602 (2004); M. Paternostro, et al., ibid. 92, 197901 (2004).

[3] J. I. Cirac, et al., Phys. Rev. Lett. 78, 3221 (1997); L. Slodička, et al., ibid. 110, 083603 (2013).

[4] L.-M. Duan and C. Monroe, Rev. Mod. Phys. 82, 1209 (2010); N. Sangouard, et al., ibid. 83, 33 (2011).

[5] H. J. Kimble, Nature (London) 453, 1023 (2008).

[6] J. M. Cai, et al., Phys. Rev. A 72, 022312 (2005); B. Bellomo, et al., ibid. 77, 032342 (2008) ; J. Maziero, et al., ibid. 80, 044102 (2009); F. F. Fanchini, et al., ibid. 81, 052107 (2010); B. Wang, et al., ibid. 81, 014101 (2010); M. M. Ali, et al., ibid. 82, 022103 (2010); J. G. Li, et al., ibid. 82, 042318 (2010); B. You and L. X. Cen, ibid. 86, 012102 (2012); B. Aaronson, et al., ibid. 88, 012120 (2013); L. Mazzola, et al., Int. J. Quantum Inform. 9, 981 (2011); T. Yu and J. H. Eberly, Science 323, 598 (2009); T. Yu and J. H. Eberly, Phys. Rev. Lett. 97, 140403 (2006); L. Mazzola, ibid. 104, 200401 (2010); J. S. $\mathrm{Xu}$, et al., Nat. Commun. 1, 7 (2010).

[7] T. Konrad, et al., Nat. Phys. 4, 99 (2008); J. S. Xu, et al., Phys. Rev. Lett. 103, 240502 (2009); O. J. Farías, et al., Science, 324, 1414 (2009).

[8] M. Nielsen, I. Chuang, Quantum Computation and Quantum Information (Cambridge Univ. Press, Cambridge, 2000).

[9] W. K. Wootters, Phys. Rev. Lett. 80, 2245 (1998).

[10] J. Yin, et al., Nature (London) 488, 185 (2012).

[11] C. H. Bennett, et al., Phys. Rev. A 54, 3824 (1996); M. Horodecki, P. Horodecki, and R. Horodecki, ibid. 60, 1888 (1999).

[12] H. Olivier and W. H. Zurek, Phys. Rev. Lett. 88, 017901 (2001); L. Henderson and V. Vedral, J. Phys. A 34, 6899 (2001).

[13] C. H. Bennett, et al., Phys. Rev. Lett. 76, 722 (1996); M. Horodecki, P. Horodecki, and R. Horodecki, ibid. 78, 574 (1997).
[14] S. Bandyopadhyay and A. Ghosh, Phys. Rev. A 86, 020304(R) (2012).

[15] A. Datta, A. Shaji, and C. M. Caves, Phys. Rev. Lett. 100, 050502 (2008); B. P. Lanyon, et al., ibid. 101, 200501 (2008); A. Shabani and D. A. Lidar, ibid. 102, 100402 (2009); D. P. DiVincenzo, et al., ibid. 92, 067902 (2004); A. Streltsov, H. Kampermann, and D. Bruß, ibid. 108, 250501 (2012); T. K. Chuan, et al., ibid. 109, 070501 (2012); J. Cui and H. Fan, J. Phys. A: Math. Theor. 43, 045305 (2010); A. Datta, Phys. Rev. A 80, 052304 (2009); Z. Merali, Nature (London) 474, 24 (2011); K. Modi, et al., Rev. Mod. Phys. 84, 1655 (2012); B. Dakić, et al., Nat. Phys. 8, 666 (2012); A. Brodutch, Phys. Rev. A 88, 022307 (2013).

[16] T. Yu, J. H. Eberly, Phys. Rev. Lett. 93, 140404 (2004); J. Laurat, et al., ibid. 99, 180504 (2007); B. Bellomo, R. Lo Franco, and G. Compagno, ibid. 99, 160502 (2007); M. P. Almeida, et al., Science 316, 579 (2007).

[17] P. Badzig, et al., Phys. Rev. A 62, 012311 (2000).

[18] S. Bandyopadhyay, Phys. Rev. A 65, 022302 (2002).

[19] F. Verstraete and H. Verschelde, Phys. Rev. Lett. 90, 097901 (2003).

[20] F. Verstraete and H. Verschelde, Phys. Rev. A 66, 022307 (2002).

[21] K. Zyczkowski, et al., Phys. Rev. A 58, 883 (1998); G. Vidal and R. F. Werner, ibid. 65, 032314 (2002).

[22] S. Luo, Phys. Rev. A 77, 042303 (2008); M. Ali, A. R. P. Rau, and G. Alber, ibid. 81, 042105 (2010); M. D. Lang and C. M. Caves, Phys. Rev. Lett. 105, 150501 (2010).

[23] Q. Chen, et al., Phys. Rev. A 84, 042313 (2011).

[24] A. Acín, et al., Phys. Rev. A 65, 052325 (2002); S. Perseguers, et al., ibid. 77, 022308 (2008); X. W. Wang and G. J. Yang, ibid. 79, 064306 (2009); J. Modławska and A. Grudka, Phys. Rev. Lett. 100, 110503 (2008); X. W. Wang, et al., Chin. Phys. Lett. 27, 100303 (2010). 